\title{
Variáveis climáticas relacionadas à poluição do ar e os efeitos causados à saúde humana
}

Air pollution related to climate variables and human health effects

\author{
Cíntia Moralles Camillol, Adriano Mendonça Souza", \\ Cláudia Aline de Souza Ramser"II
}

\begin{abstract}
RESUMO
A poluição do ar é um tema preocupante, devido aos danos que causam à saúde humana. A pesquisa teve como objetivo determinar o inter-relacionamento entre as variáveis poluentes, Partículas Totais em Suspensão (PTS), Partículas Inaláveis $\left(\mathrm{PM}_{10}\right)$, Dióxido de Enxofre $\left(\mathrm{SO}_{2}\right)$, Dióxido de Nitrogênio $\left(\mathrm{NO}_{2}\right)$, Ozônio $\left(\mathrm{O}_{3}\right)$ e Dióxido de Carbono $\left(\mathrm{CO}_{2}\right)$ e climáticas, temperatura do ar (TA), umidade relativa do ar (UR) e precipitação (PREC) no período de 1995 a 2010; nas capitais Recife/PE, São Paulo/SP, Rio de Janeiro/RJ, Belo Horizonte/BH, Porto Alegre/RS, Salvador/BA, Curitiba/PR e Vitória/ES. Foi realizada a análise de agrupamentos e fatorial, para determinar as variáveis relevantes no estudo. Em relação aos poluentes, como principais resultados, identificaram-se dois fatores, o primeiro representado por $\mathrm{CO}_{2}$ explicando $52,63 \%$ e o segundo por PTS explicando 23,22\%. Incluindo as variáveis climáticas, encontraram-se três fatores, primeiro, derivados de $\mathrm{O}_{3}$ com 44,83\%, o segundo clima caracterizado pela PREC, seguida do $\mathrm{SO}_{2}$ com 25,96\%, o terceiro, Poluentes de PTS e $\mathrm{NO}_{2}$ explicou 12,87\%. A Técnica Multivariada proporcionou uma análise conjunta dos fatores poluentes e das variáveis climáticas de modo que variáveis subjacentes fossem determinadas pela estrutura de correlação, assim evidenciando que as condições climáticas influenciam na poluição atmosférica.
\end{abstract}

Palavras-chave: Análise Multivariada; Poluição Atmosférica; Clima

\begin{abstract}
Air pollution is a matter of concern because of the damage they cause to human health. The research determined the interrelationship between the pollutant variables, Total Suspended Particles (PTS), Inhalable Particles (PM10), Sulfur Dioxide (SO2), Nitrogen Dioxide (NO2), Ozone (O3) and Carbon Dioxide (CO2) and climatic conditions, air temperature (TA), relative air humidity (RH) and precipitation (PREC) in the period from 1995 to 2010; in the capitals Recife / PE, São Paulo / SP, Rio de Janeiro / RJ, Belo Horizonte / BH, Porto Alegre / RS, Salvador / BA, Curitiba / PR and Vitória / ES. The grouping and factorial analysis were performed to determine the relevant variables in the study. Regarding the pollutants, two factors were identified, the first represented by $\mathrm{CO} 2$ explaining $52.63 \%$; the second by PTS explaining $23.22 \%$. Including the climatic variables, three factors were found, first, 03 derivatives with $44.83 \%$, the second climate characterized by PREC, followed by SO2 with $25.96 \%$, the third, PTS and NO2 Pollutants explained $12,87 \%$. The Multivariate Technique provided a joint analysis of the pollutants and climatic variables so that underlying variables were determined by the correlation structure, thus evidencing that the climatic conditions influence atmospheric pollution.
\end{abstract}

Keywords: Multivariate Analysis; Atmospheric Pollution; Climate 


\section{INTRODUÇÃO}

A Revolução Industrial acarretou várias consequências a humanidade, uma das implicações refere-se a poluição, que causa problemas para a saúde e para o meio ambiente. Com a evolução tecnológica a emissão de poluição aumentou, na área industrial e urbana, tornando-se um problema mundial. Nas grandes cidades, a poluição do ar transformou-se em uma grave ameaça à qualidade de vida da população, em particular, para idosos e crianças (TESTA, 2015).

Segundo Arbex et al. (2012) as grandes concentrações humanas nas cidades aumentaram a emissão de poluentes, o acúmulo de lixo, o volume de esgotos e o tráfego de veículos, causando doenças, como: problemas respiratórios, cardíacos, alergias, irritações e câncer, resultando prejuízos à qualidade de vida da população exposta. Os efeitos dos poluentes à saúde das pessoas podem ser agudos ou crônicos, manifestando-se após exposição ou até mesmo depois de anos ou décadas.

Estimativas globais sugerem que a poluição ambiental externa cause 1,15 milhões de óbitos em todo o mundo (correspondendo a cerca de $2 \%$ do total de óbitos) e seja responsável por 8,75 milhões de anos vividos a menos ou com incapacidade, segundo World Health Organization (2009), enquanto que a poluição no interior dos domicílios cause aproximadamente 2 milhões de óbitos prematuros e 41 milhões de anos vividos a menos ou com incapacidade. Para o Brasil, a OMS (2018) estimou que a poluição atmosférica causou cerca de 20 mil óbitos/ano, valor cinco vezes superior ao número de óbitos ocasionados pelo tabagismo ambiental/passivo e 10,7 mil óbitos/ano decorrentes da poluição do ar em ambientes internos.

No Brasil, assim como em diversos países, existem padrões de qualidade do ar, estabelecendo limites de tolerância, para assegurar que a população tenha qualidade de vida. Como resposta a problemas enfrentados referentes à poluição, o Ministério da Saúde (MS), por meio da Coordenação Geral de Vigilância em Saúde Ambiental, estruturou, a partir de 2001, o Programa de Vigilância em Saúde Ambiental Relacionada à Qualidade do Ar (Vigiar).

Os poluentes são representados por Partículas Totais em Suspensão (PTS), Partículas Inaláveis $\left(\mathrm{PM}_{10}\right)$, Dióxido de Enxofre $\left(\mathrm{SO}_{2}\right)$, Dióxido de Nitrogénio $\left(\mathrm{NO}_{2}\right)$, 
Ozônio $\left(\mathrm{O}_{3}\right)$ e Dióxido de Carbono $\left(\mathrm{CO}_{2}\right)$ e as variáveis condições climáticas: temperatura do ar (TA), umidade relativa do $\operatorname{ar}(\mathrm{UR})$ e precipitação (PREC). Este estudo aborda um assunto atual que atinge parte significativa da população, partindo do pressuposto que todos, na sociedade, têm a responsabilidade de contribuir com o processo de mudança para um novo padrão de comportamento frente aos recursos naturais, que são elementos da natureza essenciais para a sobrevivência do ser humano. O tema em estudo é de relevância para a sociedade, uma vez que essa abordagem permite uma reflexão profunda sobre os efeitos dos poluentes à saúde das pessoas, propiciando mudanças de comportamento e amadurecimento sobre a temática no seio da sociedade em geral.

Assim a pesquisa tem como objetivo determinar o inter-relacionamento entre poluentes e variáveis climáticas nas principais capitais brasileiras, por meio da análise de agrupamentos e da análise fatorial, identificando as variáveis mais relevantes no conjunto de dados.

\section{POLUIÇÃO ATMOSFÉRICA}

Dentre os diferentes tipos de poluição observados no meio ambiente, a contaminação do ar atmosférico tem sido tema de constante discussão e estudos científicos nas últimas décadas. A poluição do ar é um fator de risco direto para a saúde humana, assim como para o ecossistema (DAPPER, SPOHR, ZANINI, 2016).

Santiago et al. (2015) conceituam o ar poluído como uma mistura de partículas, material particulado (MP10) e gases de partículas sólidas (poeiras, fuligens e fumaças), que são emitidos para a atmosfera, principalmente, por indústrias, veículos automotivos, termoelétricas, queima de biomassa e de combustíveis fósseis.

Conforme o Conselho Nacional do Meio Ambiente (CONAMA, 1990), os poluentes podem ser divididos em duas categorias, os primários, que são emitidos diretamente pelas fontes de poluição, e os secundários, que são formados na atmosfera, através da reação química entre poluentes primários e os constituintes naturais da atmosfera.

Ainda o conselho CONAMA (1990), a determinação sistemática da qualidade do ar, fontes e efeitos, de alguns poluentes, dá-se pela medição dos seguintes 
parâmetros: Material Particulado Total em Suspensão (PTS), Fumaça e Partículas Inaláveis (PM10), que representam o material sólido emitido pelas fontes poluidoras ou em suspensão na atmosfera (poeira, fumaça, fuligem); Dióxido de Enxofre (SO2), que está relacionado com o uso de combustíveis de origem fóssil, contendo enxofre, é emitido por veículos ou instalações industriais; Monóxido de Carbono (CO), que está relacionado ao processo de combustão em fontes móveis, motores à gasolina, diesel ou álcool, quanto a fontes fixas industriais; Ozônio (03), que é um gás invisível, com cheiro marcante, composto por três átomos de oxigênio, altamente reativo e que está presente na alta atmosfera e na superfície. Quando presente nas altas camadas da atmosfera protege-nos dos raios ultravioletas do sol e, quando formado próximo ao solo, comporta-se como poluente; Dióxido de Nitrogênio (NO2), que é formado pela reação do óxido de nitrogênio e do oxigênio reativo, presentes na atmosfera.

A concentração de substâncias tóxicas no organismo humano pode trazer sérias consequências, como doenças respiratórias, cardíacas e até mesmo a morte. A literatura biomédica está repleta de estudos sobre os efeitos agudos da poluição do ar sobre a saúde, com ênfase em dois desfechos: mortalidade e internações hospitalares, segundo Brunekreef e Holgate (2002).

A partir do compromisso pela qualidade do ar e saúde ambiental, o Governo Federal do Brasil assumiu a responsabilidade de promover a reflexão sobre as necessidades e os desafios desse tema como parte integrante de um Plano Nacional de Qualidade do $\operatorname{Ar}$ (PNQA). O objetivo é "proteger o meio ambiente e a saúde humana dos efeitos da contaminação atmosférica, por meio da implantação de uma política contínua e integrada de gestão da qualidade do ar no país" controlado pelo Ministério do Meio Ambiente (CONAMA, 2018).

\section{MATERIAIS E MÉTODOS}

Os poluentes utilizados para a realização desta pesquisa são PTS, PM10, SO2, NO2, O3, CO2 obtidos no site do IBGE (2016), do ano de 1995 a 2010, das cidades: Recife/PE, São Paulo/SP, Rio de Janeiro/RJ, Belo Horizonte/BH, Porto Alegre/RS, Salvador/BA, Curitiba/PR e Vitória/ES. 
As variáveis climáticas, a temperatura do ar (TA), a umidade relativa do ar (UR) e a precipitação pluviométrica (PREC) foram coletadas no site ICEA (2016) (Sistema de Geração e Disponibilização de Informações Climatológicas), no mesmo período amostral. A variável PREC foi incluída como variável explicativa para verificar a sua influência nas demais variáveis e compreender a sua relação na poluição do ar.

$\mathrm{Na}$ Tabela 1 são apresentados os dados referentes à localização de cada estação meteorológica do estudo, disponibilizados pelo site do ICEA (2016).

Tabela 1 - Caracterização das estações meteorológicas

\begin{tabular}{lccccc}
\hline Cidade & Estação & \multicolumn{1}{c}{ Altitude } & Latitude & Longitude & Fuso \\
\hline Belo Horizonte/MG & Pampulha & 77 & 19515 & $4357 \mathrm{~W}$ & 3 \\
Curitiba/PR & Afonso Pena & 910 & 25315 & $4911 \mathrm{~W}$ & 3 \\
Porto Alegre/RS & Porto Alegre & 4 & 30005 & $3111 \mathrm{~W}$ & 3 \\
Recife/PE & Dos Guararapes & 11 & 08085 & $3455 \mathrm{~W}$ & 3 \\
Rio de Janeiro/RJ & Santos Dumont & 3 & 22545 & $4310 \mathrm{~W}$ & 3 \\
Salvador/BA & Dep. Luis Eduardo Magalhães & 13 & 12545 & $3820 \mathrm{~W}$ & 3 \\
São Paulo/SP & Congonhas & 789 & 23375 & $4639 \mathrm{~W}$ & 3 \\
Vitória/ES & Eurico de Aguiar Salles & 3 & 20155 & $4017 \mathrm{~W}$ & 3 \\
\hline
\end{tabular}

Fonte: ICEA (2016)

O estudo foi realizado considerando-se as variáveis poluentes e, num segundo momento, incluíram-se, na análise, as variáveis climáticas para detectar o interrelacionamento entre elas. Inicialmente, por meio da estatística descritiva, verificamse a amplitude atribuída às variáveis poluentes e às condições climáticas, bem como homogeneidade em relação à média, à mediana, ao desvio padrão e ao coeficiente de variação.

Destaca-se que, antes de realizar qualquer procedimento em termos de análise multivariada, as variáveis foram padronizadas, seguindo-se, neste caso, a distribuição normal. Dessa forma, cada variável deve ser capaz de apresentar o mesmo peso de ponderação sem que os efeitos das unidades de medidas influenciem o estudo.

Na etapa da análise de agrupamento, Vicini, Souza e Souza (2018) adotaram o processo de aglomeração hierárquica, utilizou-se a distância euclidiana com métrica e o método de aglomeração de Ward. A ideia básica consistiu em colocar, em um mesmo grupo, objetos que sejam similares de acordo com algum critério prédeterminado. 
Para Análise Fatorial Tino (2005) e King (2008) mensuraram as variáveis, e identificaram as quais apresentam a mesma estrutura subjacente. E com a análise dos componentes principais, reduziram a quantidade de variáveis observadas a um número de fatores, e os fatores representam as dimensões que resumem e explicam o conjunto de variáveis originais

Tabachnick, Fidell e Ullman (2007), para a seleção dos fatores recomendam o uso de autovalores superiores a 1 (um) e/ou variância acumulada superior a 70\%. E para etapa de realização da AF os autores ressaltam que se devem verificar a adequabilidade da base de dados; determinando a técnica de extração e o número de fatores extraídos; decidiram-se o tipo de rotação dos fatores.

O modelo fatorial, segundo Vicini, Souza e Souza (2018), foi definido como segue na equação (1)

$$
X_{i}=a_{i 1} F_{1}+a_{i 2} F_{2}+a_{i 3} F_{3}+\ldots+a_{i m} F_{m}+e_{i}
$$

Onde:

$X_{i}$ : variáveis observadas, $i=1,2, \ldots, p ;$

$\mathrm{a}_{\mathrm{ij}}$ : cargas fatoriais associadas ao j-ésimo fator na i-ésima variável;

$F_{m}$ : fatores comuns, $j=1,2, \ldots, m$;

ei: erro que capta a variação não explicada pela combinação linear, específica da variável $X_{i} ;$

A extração de fatores forma uma combinação linear das variáveis originais ou da matriz de correlações, elas serão combinadas para formar um fator, e assim, sucessivamente, com todas as demais variáveis da matriz de correlação. E a combinação linear entre variáveis pode ser assim definida (equação2) (VICINI, SOUZA e SOUZA, 2018):

$F_{j}=W_{j 1} X_{1}+W_{j 2} X_{2}+W_{j 3} X_{3}+\ldots+W_{j p} X_{p}$

Sendo:

Fj: estimativa (escore) do j-ésimo fator comum; 
$W_{j}$ : peso ou coeficiente do escore fatorial;

$X_{i}$ : i-ésima variável de decisão (variável independente);

p: número de variáveis de decisão (variáveis independentes)

Caso existam fatores que apresentem variáveis com o mesmo grau de informação representado por cargas fatoriais significativas, realiza-se uma rotação Varimax Normalizada.

Segundo Vicini, Souza e Souza (2018), a ideia básica da Rotação Varimax Normalizada é identificar alguns fatores que possuam variáveis que tenham alta correlação e outros com variáveis que possuam baixa correlação. A matriz de correlação entre variáveis foram calculadas pelo coeficiente de correlação de Pearson representado na Equação 3.

Coeficiente de Pearson

$$
\rho=\frac{\sum_{1}^{n}=1\left(\chi_{i}-\bar{\chi}\right)\left(\gamma_{1}-\bar{\gamma}\right)}{\sqrt{\sum_{1=1}^{n}\left(\chi_{i}-\bar{\chi}\right)^{2} \sqrt{\sum_{1=1}^{n}\left(\gamma_{i}-\bar{\gamma}\right)^{2}}}}
$$

Considerando dois vetores aleatórios $\chi$ e $\gamma$ de tamanhos $n$ com médias $\bar{\chi}$ e $\bar{\gamma}$ respectivamente.

Com base nas etapas metodológicas foi possível alcançar os objetivos do estudo e, compreender o inter-relacionamento das variáveis de modo simultâneo.

\section{Resultados e Discussões}

Na Tabela 2, apresentam-se os resultados da análise estatística descritiva dos dados, no período entre 1995 a 2010.

Tabela 2 - Principais resultados da análise estatística descritiva dos poluentes

\begin{tabular}{lcccc}
\hline Variáveis & Média & Mediana & Desvio Padrão & Coef. De Variação \\
\hline PTS & 0,02 & $-0,75$ & 1,00 & 50 \\
$\mathrm{PM}_{10}$ & 0,01 & $-0,08$ & 1,00 & 100 \\
$\mathrm{SO}_{2}$ & $-0,01$ & $-0,27$ & 0,99 & -99 \\
$\mathrm{NO}_{2}$ & 0,01 & $-0,19$ & 1,00 & 100 \\
$\mathrm{O}_{3}$ & 0,01 & $-0,13$ & 0,99 & 99 \\
$\mathrm{CO}_{2}$ & 0,01 & $-0,09$ & 1,00 & 100 \\
\hline
\end{tabular}


Conforme Tabela 2, os poluentes possuem um coeficiente de variação elevado exceto o PTS, portanto esses valores oscilam ao longo do período analisado. Os coeficientes de variações resultam em uma amostra heterogenia. O PTS é o valor com menor dispersão. Com base nos estudos de, Evo et al. (2011); Nascimento e Carvalho (2013); Nardocci et al (2013); Silva et al. (2013); Paiva (2014); Barbosa et al (2015); Roman et al (2015); Tuan et al (2015), observaram que as variáveis poluentes resultaram o coeficiente de variação alto, indicando alta dispersão relativa dos valores.

Na Tabela 3, as médias anuais das variáveis climáticas em relação às capitais estudadas.

Tabela 3 - Médias anuais da umidade relativa do ar (UR), precipitação (PREC) e temperatura do ar (TA)

\begin{tabular}{lccc}
\hline Cidade & $\begin{array}{c}\text { Umidade Relativa } \\
\text { do } \operatorname{Ar}(\mathrm{UR})\end{array}$ & $\begin{array}{c}\text { Precipitação } \\
\text { (PREC) }\end{array}$ & $\begin{array}{c}\text { Temperatura } \\
\text { do Ar (TA) }\end{array}$ \\
\hline Vitória & $82,37(2001)$ & $1,82(2005)$ & $26,30(1998)$ \\
Porto Alegre & $85,30(2010)$ & $1,50(2003)$ & $20,50(2001)$ \\
Curitiba & $88,80(1999)$ & $2,05(1999)$ & $18,00(2002)$ \\
São Paulo & $82,70(1996)$ & $1,87(2009)$ & $21,00(2001)$ \\
Belo Horizonte & $72,50(2006)$ & $1,86(1995)$ & $23,00(1998)$ \\
Salvador & $83,00(1999 / 2000 / 2009)$ & $2,12(1999)$ & $28,00(1995)$ \\
Rio de Janeiro & $82,70(2009)$ & $1,76(2010)$ & $24,50(1998)$ \\
Recife & $79,00(2000)$ & $3,84(2000)$ & $29,00(1998)$ \\
\hline
\end{tabular}

Na Tabela 3, foram listados os anos em que ocorreram elevação nas médias da umidade relativa do ar (UR), precipitação (PREC) e temperatura do ar (TA). A PREC, no caso de Salvador, Curitiba e Recife, as médias anuais foram elevadas nos anos de 1999, 1999, 2000, respectivamente. Salvador e Recife referiram-se as médias de temperatura do ar (TA) mais altas das cidades estudadas, nos anos de 1995 e 1998. Segundo a Organização Mundial de Saúde (OMS, 2018), o nível ideal da UR para o organismo humano é entre $40 \%$ e $70 \%$. Acima desses valores, o ar fica praticamente saturado de vapor d'água, o que interfere no mecanismo humano de controle da temperatura corporal, exercido pela transpiração, em todas as cidades o nível de UR foi bem maior que o indicado pela OMS. 
A temperatura do ar constituiu um parâmetro de interesse para o estudo da dispersão de poluentes, visto que temperaturas mais elevadas conduzem à formação de movimentos verticais ascendentes mais pronunciados (convecção), gerando um eficiente arrastamento dos poluentes localizados dos níveis mais baixos para os níveis mais elevados. Por outro lado, temperaturas mais baixas não induzem aos movimentos verticais termicamente induzidos, o que permite a manutenção de poluentes atmosféricos em níveis mais baixos, confirmam Damilano e Jorge (2006).

Segundo os dados do IBGE (2016), existe um grande número de morbidades causado por doenças respiratórias principalmente em São Paulo/SP, seguido pela cidade do Rio de Janeiro. A OMS (2018) divulgou a perda precoce de cerca de oito milhões de vidas no mundo pela poluição do ar. Dessas, cerca de 3,7 milhões devem ser relacionadas à poluição do ar externa, o que representa $46 \%$ de todas as fontes de poluentes relacionadas ao ar.

Seguindo a análise, identificaram-se as variáveis pertencentes ao mesmo agrupamento, ilustrados na Figura 1, o dendrograma foi constituído com todas as variáveis, os poluentes e as condições climáticas do período de 1995 a 2010 com método de aglomeração de Ward e métrica da distância euclidiana.

Figura 1 - Dendrograma envolvendo as variáveis poluentes e condições climáticas

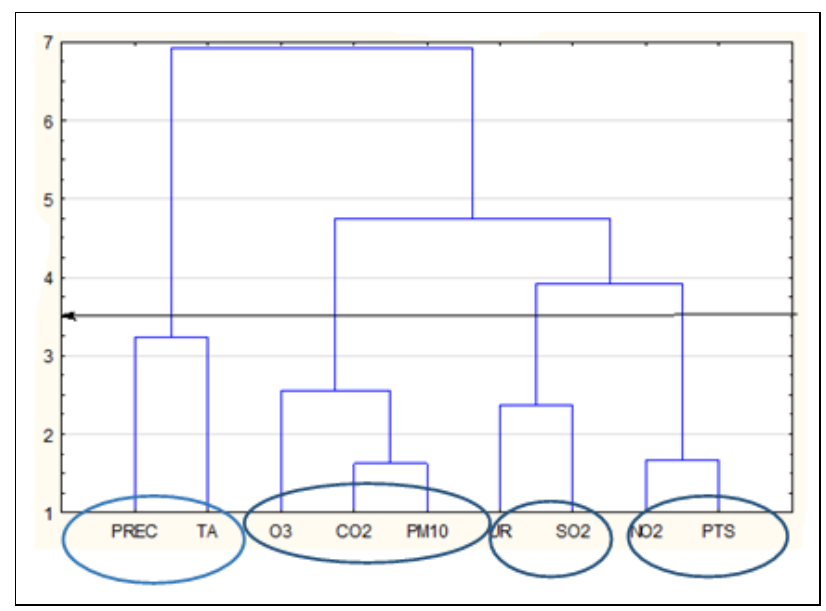

Na Figura 1 observou-se a formação de quatro grupos, representados pelas variáveis de maior relevância dentro do conjunto. O primeiro agrupamento foi formado pelas variáveis precipitação (PREC) e temperatura do ar (TA); o segundo, por 
ozônio (O3), dióxido de carbono (CO2) e partículas de inaláveis (PM10); o terceiro, pela umidade relativa do ar (UR) e dióxido de enxofre (SO2); e o quarto grupo, pelos poluentes, dióxido de nitrogênio (NO2) e partículas totais em suspensão (PTS).

O dendrograma representou a associação da precipitação com a temperatura, formando, assim, o primeiro grupo por similaridade. Dependendo da região das cidades citadas no estudo, a precipitação (chuvas, neve, granizo) e a temperatura (elevadas, amenas ou baixas) influenciaram diretamente uma na outra.

No segundo grupo, foram formados por as partículas inaláveis (PM10), que são emitidas principalmente pelos veículos a Diesel, assim como o dióxido de carbono (CO2), responsável pela combustão e pelo efeito estufa, e o ozônio (O3), que está presente nas zonas urbanas e é responsável pela radiação ultravioleta (UV).

A umidade do ar é responsável pela formação das nuvens e está diretamente relacionada com a água. Mas quando não há umidade, o ar está seco, isto é, isento de água e composto por gases. Formaram o terceiro grupo, a umidade relativa do ar (UR) e o dióxido de carbono (SO2), uniram-se por similaridade.

No último grupo, o poluente dióxido de nitrogênio (NO2), e o PTS (partícula em suspensão), responsáveis por a combustão na atmosfera. Logo, os poluentes e as variáveis climáticas apresentaram uma aproximação de ocorrência. Após o diagnóstico da similaridade das variáveis, desenvolveu-se a Análise Fatorial determinando os autovalores e o percentual de explicação de cada fator, entre outros resultados (Tabela 4), conforme recomendado por Hair et al. (1998).

Tabela 4 - Autovalores e percentual da variância explicada de cada componente das variáveis poluentes

\begin{tabular}{lcccc}
\hline \multicolumn{5}{c}{ Autovalores } \\
$\begin{array}{l}\text { Número de } \\
\text { componentes }\end{array}$ & Autovalores & $\begin{array}{c}\text { Percentual da } \\
\text { variância explicada }\end{array}$ & $\begin{array}{c}\text { Autovalores } \\
\text { acumulados }\end{array}$ & $\begin{array}{c}\text { Percentual da variância } \\
\text { explicada acumulada }\end{array}$ \\
\hline 1 & 3,16 & 52,63 & 3,15 & 52,63 \\
2 & 1,39 & 23,22 & 4,55 & 75,86 \\
3 & 0,97 & 16,19 & 5,52 & 92,05 \\
4 & 0,38 & 6,36 & 5,90 & 98,42 \\
5 & 0,09 & 1,54 & 5,99 & 99,96 \\
6 & 0,002 & 0,03 & 6,00 & $100, .00$ \\
\hline
\end{tabular}


Observou-se que os dois primeiros valores representaram cerca de 75,8612\% da variância explicada acumulada. Portanto, as variáveis foram resumidas pelos dois primeiros componentes principais. Pode-se também fazer essa seleção por um critério sugerido por Kaiser (1960), onde se incluem somente os componentes com valores próprios maiores do que 1 (um). Neste caso, resultou-se em dois autovalores.

Na Tabela 5, verificaram-se o resultado da extração dos fatores por meio da técnica de componentes principais com rotação Varimax Normalizada para os poluentes.

Tabela 5- Variáveis poluentes de maior peso em relação aos fatores após rotação Varimax Normalizada dos poluentes

\begin{tabular}{|c|c|c|}
\hline \multirow[b]{2}{*}{ Variáveis } & \multicolumn{2}{|l|}{ Fatores } \\
\hline & Fator 1 & Fator 2 \\
\hline PTS & $-0,006$ & $0,82^{*}$ \\
\hline $\mathrm{PM}_{10}$ & 0,30 & 0,23 \\
\hline $\mathrm{SO}_{2}$ & 0,07 & 0,20 \\
\hline $\mathrm{NO}_{2}$ & 0,10 & $0,94 *$ \\
\hline $\mathrm{O}_{3}$ & $0,96 *$ & $-0,001$ \\
\hline $\mathrm{CO}_{2}$ & 0,63 & 0,34 \\
\hline
\end{tabular}

Na Tabela 5, observaram-se os fatores e, são consideradas as variáveis com cargas fatoriais superiores 0,7 valor estipulado como ponte de corte, considerando que elas fossem estatisticamente significativas. Ainda concluiu-se que o fator 1 (um) derivou do maior autovalor que foi 3,16 e a carga fatorial foi 0,96 , representado pelo poluente 03. O fator 2 (dois) derivou de um autovalor de 1,39 e explicado por duas variáveis de maiores cargas fatoriais 0,94 , representado pelo poluente NO2 e de 0,82 representado pelo poluente PTS.

Para a visualização desses fatores estudados, optaram-se pela utilização dos planos fatoriais representando-os 1 (um) e 2 (dois). 
Figura 2 - Relação entre os fatores 1 (um) e 2 (dois), poluentes em suspensão e poluentes derivados de $\mathrm{CO}_{2}$ com rotação Varimax Normalizada

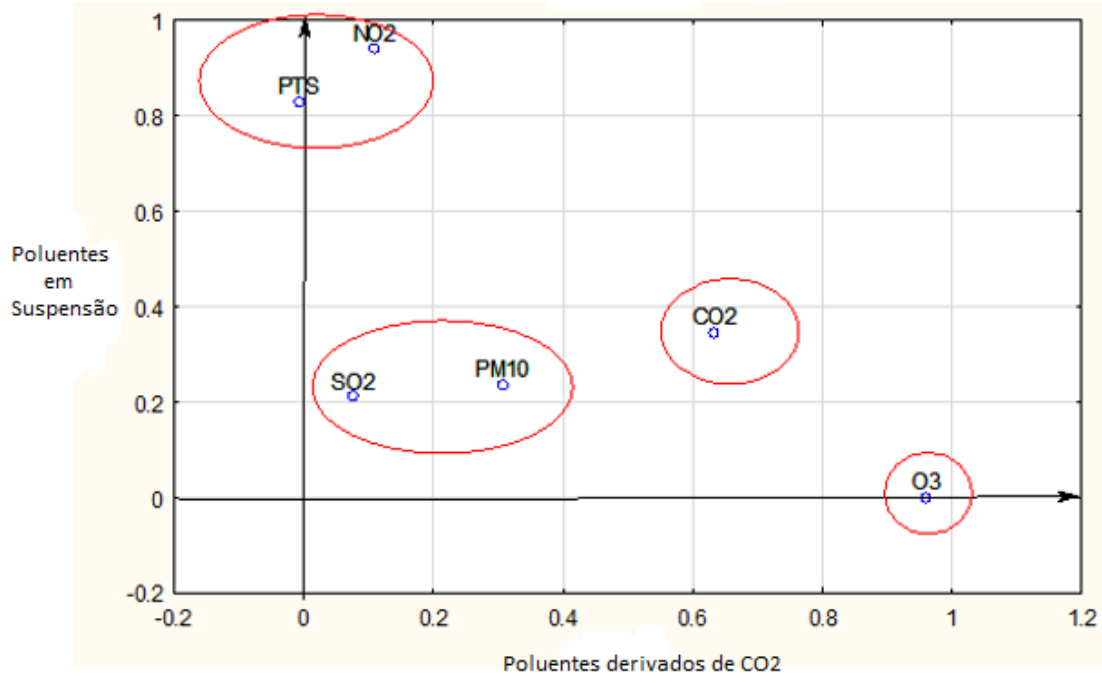

Observou-se a formação de quatro grupos por similaridade de explicação, isto é, agrupadas por fatores, a variável que está mais distante da origem, no eixo $X$, foi o poluente $\mathrm{O} 3$ e, no eixo $\mathrm{Y}$, as variáveis PTS e NO2, foram as que melhor explicaram e que foram mais representativas. Ainda destacaram-se o poluente $\mathrm{CO} 2$, que ficou longe da origem, mais longe dos eixos. As variáveis SO2 e PM10 resultaram em baixa representatividade, pois, localizaram-se próximas à origem do plano fatorial (Figura 2). Realizou-se a análise fatorial, incluindo, além dos poluentes, as variáveis climáticas como: temperatura do ar (TA), umidade relativa do ar (UR) e precipitação (PREC), para concluírem o comportamento do sistema de variáveis.

Tabela 6 - Autovalores e percentual da variância explicada de cada componente das variáveis poluentes e climáticas

Autovalores

Extração dos componentes principais

\begin{tabular}{lcccc}
\hline $\begin{array}{l}\text { Número de } \\
\text { componente }\end{array}$ & Autovalores & Percentual da variância explicada & $\begin{array}{c}\text { Autovalores } \\
\text { acumulados }\end{array}$ & $\begin{array}{c}\text { Percentual da variância } \\
\text { explicada acumulada }\end{array}$ \\
\hline 1 & 4,034770 & 44,83078 & 4,034770 & 44,8308 \\
2 & 2,337105 & 25,96783 & 6,371875 & 70,7986 \\
3 & 1,158808 & 12,87564 & 7,530683 & 83,6743 \\
4 & 0,728703 & 8,09670 & 8,259386 & 91,7710 \\
5 & 0,524487 & 5,82763 & 8,783873 & 97,5986 \\
6 & 0,210397 & 2,33774 & 8,994270 & 99,9363 \\
7 & 0,005730 & 0,06367 & 9,000000 & 100,0000 \\
\hline
\end{tabular}


Analisou-se todas as variáveis do estudo (poluentes e condições climáticas), resultando 83,6743\% de variância explicada acumulada (Tabela 6). Portanto, as variáveis foram resumidas pelos três primeiros componentes principais. Ainda foi possível observar que os três primeiros fatores possuem autovalores 4,034770; 2,337105 e 1,158808, que corresponderam a 44,83\%; $25,967 \%$ e 12,87\% da variância explicada.

Na Tabela 7, verificou-se o resultado da extração dos componentes principais, realizado pela Rotação Varimax Normalizada, para todas as variáveis do estudo, poluentes e condições climáticas.

Tabela 7 - Composição dos fatores selecionados com rotação Varimax Normalizada para as variáveis poluentes e condições climáticas

\begin{tabular}{llll}
\hline & \multicolumn{3}{l}{ Fatores com Rotação Varimax Normalizada } \\
\cline { 2 - 4 } Variáveis & Fator 1 & Fator 2 & Fator 3 \\
\hline PTS & -0.043099 & 0.006473 & 0.843129 \\
PM10 & 0.332984 & -0.008245 & 0.254876 \\
SO2 & 0.143546 & 0.945086 & 0.172223 \\
NO2 & 0.136319 & 0.290675 & 0.926785 \\
O3 & 0.969540 & 0.043589 & 0.010762 \\
CO2 & 0.598112 & -0.356483 & 0.386213 \\
TA & -0.118981 & -0.203163 & -0.222217 \\
UR & 0.252378 & 0.397426 & 0.149408 \\
PREC & 0.167084 & -0.960406 & -0.090319 \\
\hline
\end{tabular}

Logo, o fator 1 (um) foi derivado do maior autovalor 4,0347770 e o poluente de maior peso, 0,969540 foi representado por 03, por sua vez, o fator 2 (dois) foi derivado de um autovalor de 2,33705 e representa o poluente SO2 e a variável climática precipitação (PREC). O fator 3 foi oriundo de um autovalor de 1,158808 e foi representado pelos poluentes PTS e NO2. Ressalta-se, em uma análise, o fator 1 (um) possuiu um grau de importância maior, pois o autovalor que ele originou, 4,0347770, foi quase o dobro do segundo autovalor 2,33705 em termos de magnitude e quase quatro vezes o valor do terceiro autovalor.

Seguindo com a análise, projetou-se o gráfico da distribuição da nuvem de variáveis, no círculo de correlações, conforme a Figura 3. Uma das utilizações do círculo unitário é a realização da sobreposição deste sobre o primeiro plano fatorial, 
sendo assim, o círculo de correlação unitário representou o comportamento das variáveis climáticas em relação às variáveis poluentes, pois quanto menor o ângulo formado pelas variáveis, maior será a influência entre elas, visto que representaram graficamente o grau de correlação entre elas.

Figura 3 - Círculo de Correlação Unitária das Variáveis Climáticas e dos Poluentes

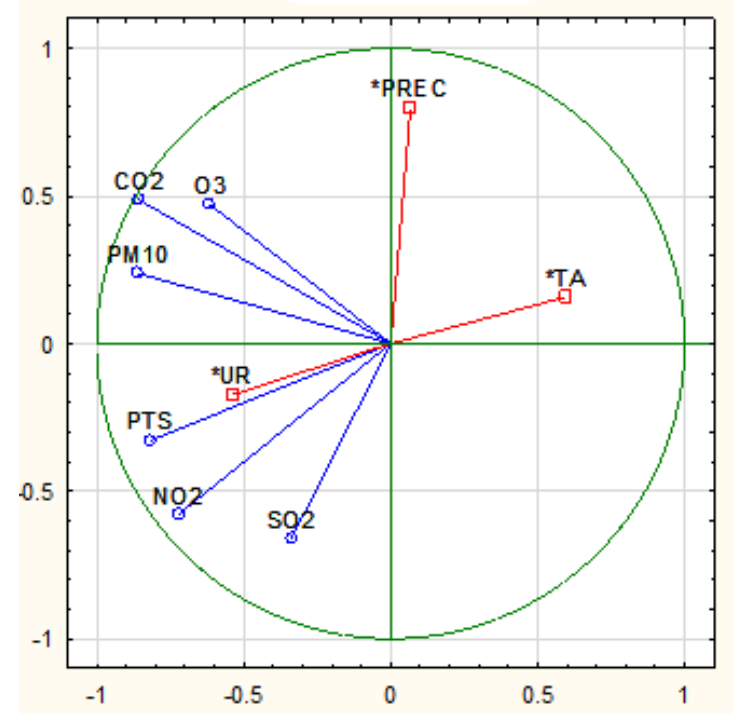

Constatou-se, na Figura 3, que nenhuma variável está sobreposta a outra, resultando, assim, que elas não possuem a mesma representatividade. Outro fato importante foi que CO2, PM10, PTS e NO2 estão bem próximas do círculo unitário, demonstrando-se, dessa forma, uma maior contribuição em relação as outras variáveis. O círculo unitário na Figura 3 formou um ângulo muito pequeno com a umidade relativa do ar (UR) e o poluente PTS, evidenciando a presença de correlação entre estas duas variáveis, logo a UR interfere na PTS; a precipitação (PREC) relacionou-se com a temperatura do ar (TA), pois o ângulo formado entre estas duas variáveis é menor do que 450; os poluentes $\mathrm{CO} 2$, O3 e PM10 foram representados no quadrante II; a umidade relativa do ar (UR) influencia os poluentes PTS, NO2 e SO2; a UR influenciou os poluentes do quadrante $\mathrm{Il}$; $\mathrm{CO} 2$ e O3 sofreram influência da precipitação (PREC); CO2 é o poluente mais distante da origem, porém o mais próximo do círculo unitário. 
Na Tabela 8, o resultado da matriz de correlação entre os poluentes PM10, CO2, O2 e a variável PREC, verificando se estão interligadas.

Tabela 8 - Resultados da Matriz de Correlação entre $\mathrm{O}_{3}, \mathrm{CO}_{2}, \mathrm{PM} 10$ e PREC

\begin{tabular}{lllll}
\hline & \multicolumn{4}{l}{ Correlação } \\
\cline { 2 - 5 } Variáveis & $\mathrm{O}_{3}$ & $\mathrm{CO}_{2}$ & $\mathrm{PM}_{10}$ & $\mathrm{PREC}$ \\
\hline $\mathrm{O}_{3}$ & 1 & 0,70 & 0,52 & 0,10 \\
$\mathrm{CO}_{2}$ & & 1 & 0,81 & 0,40 \\
$\mathrm{PM}_{10}$ & & & 1 & 0,05 \\
$\mathrm{PREC}$ & & & & 1 \\
\hline
\end{tabular}

Com a matriz de correlação da Tabela 8 foi possível observar que existe uma correlação forte entre as variáveis CO2 e PM10, e ainda uma correlação moderada entre $\mathrm{CO} 2$ e 03. Sendo assim, concluiu-se que a variável CO2 está interligada com o poluente $\mathrm{O} 3$ e PM10.

Já na Tabela 9, o resultado da matriz de correlação entre os poluentes PTS, NO2 e SO2 e a variável UR, verificando se estão interligadas entre elas.

Tabela 9- Resultados da Matriz de Correlação entre UR, PTS, $\mathrm{NO}_{2}$ e $\mathrm{SO}_{2}$

\begin{tabular}{lllll}
\hline & \multicolumn{4}{l}{ Correlação } \\
\cline { 2 - 5 } Variáveis & UR & PTS & $\mathrm{NO}_{2}$ & $\mathrm{SO}_{2}$ \\
\hline UR & 1 & 0,47 & 0,27 & 0,59 \\
PTS & & 1 & 0,80 & 0,22 \\
$\mathrm{NO}_{2}$ & & & 1 & 0,47 \\
$\mathrm{SO}_{2}$ & & & & 1 \\
\hline
\end{tabular}

Observou-se, na Tabela 9, por meio da matriz de correlação, que os poluentes PTS e NO2 resultaram em uma forte correlação entre si, indicando que essas variáveis estão interligadas. Sabe-se assim que as variáveis poluentes e climáticas interferem na saúde humana, principalmente os poluentes.

Resultados apresentados por d'Amato et al. (2011) assinalam que a poluição atmosférica está relacionada com o aumento da morbilidade e mortalidade respiratória e cardiovascular, principalmente devido às alterações provocadas pela constante exposição aos elevados níveis de poluição que promovem a disrupção da 
barreira mucociliar, promovendo a inflamação das vias aéreas e a facilitação das respostas inflamatórias primárias induzidas pelos alergênicos, devido ao aumento da penetração e do contato com as células do sistema imunitário. Os autores ainda afirmam que os poluentes $\mathrm{O} 3, \mathrm{NO} 2, \mathrm{SO} 2, \mathrm{PM}$ associados a áreas urbanas e de intenso tráfego automóvel são responsáveis por esses problemas respiratórios.

Autores como Evo et al. (2011); Nascimento e Carvalho (2013); Nardocci et al (2013); Silva et al. (2013); Paiva (2014); Barbosa et al (2015); Roman et al (2015); Tuan et al (2015); observaram que a principal patologia relacionada com a poluição do ar refere-se a doenças respiratórias, havendo o agravante das variáveis: temperatura do ar e umidade relativa do ar. Os poluentes que tiveram maior destaque no estudo da revisão sistemática foram: dióxido de nitrogênio (NO2), dióxido de carbono (CO2), partículas inaláveis (PM10), partículas em suspensão (PTS), ozônio (O3) e o dióxido de enxofre (SO2).

Desse modo classificando a morbidade no aparelho respiratório, a cidade de São Paulo/SP aparece em primeiro lugar, com uma maior taxa de portadores de doenças respiratórias, seguida pelo Rio de Janeiro/RJ.

\section{CONCLUSÃO}

Para realização da pesquisa, uma das limitações foi a dificuldade em obter os dados para o estudo, porque o site IBGE (2016) não disponibiliza dados recentes sobre a poluição atmosférica nas capitais utilizadas, sendo que não há dados disponíveis para outras cidades.

Realizou-se a estatística descritiva com os dados do IBGE (2016); do ano de 1995 a 2010, verificou-se que os poluentes PTS e PM10 seguidos pelos poluentes SO2 e CO2 são os que mais se destacam e merecem uma maior atenção, apresentando um coeficiente de variação mais elevado do que os demais.

A análise multivariada confirmou que os poluentes do estudo em questão contribuem muito para a poluição atmosférica desde o ano de 1995. A análise de agrupamento identifica concomitantemente as variáveis pertencentes a cada grupo de acordo com a literatura. 
A análise fatorial identificou dois fatores como os mais importantes com uma explicação de $75,86 \%$, quando analisa somente os poluentes. Ao avaliar todas as variáveis (poluentes e condições climáticas), identificou-se três fatores com uma explicação de 83,6743\%. Do círculo de correlação unitária das variáveis, rotacionada para todas as variáveis do estudo, a precipitação relacionou-se com a temperatura do ar, pois o ângulo formado entre as variáveis é menor do que $90^{\circ}$ e essas variáveis estão localizadas no primeiro quadrante.

Os poluentes do segundo quadrante possuem um ângulo menor de $90^{\circ}$, sofrendo influência da umidade do ar que está no terceiro quadrante, por formar um ângulo menor que $90^{\circ}$. Verificou-se que as variáveis CO2 e O3 no segundo quadrante sofrem influência da precipitação e que CO2 é o poluente que melhor representa o fator 1 (um) em relação ao fator 2 (dois). Neste sentido, a variável está bem próximo ao círculo unitário, o que configura como o poluente é mais representativo.

Já no terceiro quadrante, a umidade do ar influência os poluentes PTS, NO2 e SO2 e consequentemente. Logo, precipitação (PREC) e umidade relativa do ar (UR) são bons índices para auxiliar na elevação ou queda desses poluentes.

Portanto a Análise Multivariada proporcionou verificar em conjunto, os fatores poluentes e das variáveis climáticas, de modo que variáveis subjacentes foram determinadas pela estrutura de correlação evidenciando que as condições climáticas influenciam na poluição atmosférica. Deste modo, sugere-se ampliar o estudo para investigar pesquisas realizadas em outros países, relacionando os efeitos da poluição do ar na saúde humana e as suas consequências nas condições climáticas. 


\section{Referências}

ARBEX MA, SANTOS UP, MARTINS LC, SALDIVA PHN, PEREIRA LAA, BRAGA ALF. A poluição do ar e o sistema respiratório. J Bras Pneumol. 2012;38(5):643-655.

BANCO DE DADOS CLIMATOLÓGICOS DA AERONÁUTICA - ICEA. Sistema de Geração e Disponibilização de Informações Climatológicas. [Internet] Comando da Aeronáutica [citado 20 junho 2016]. Disponível em: http://clima.icea.gov.br/clima/.

BARBOSA S, FARHAT S, MARTINS L, PEREIRA L, SALDIVA P, ZANOBETTI A, et al. Poluição do ar e a saúde das crianças: a doença falciforme. Cadernos de Saúde Pública 2015;31(2): 265-75.

BRUNEKREEF B, HOLGATE ST. Air pollution and health. The lancet 2002;360(9341):1233-1242.

CESAR ACG, NASCIMENTO LFC, CARVALHO Jr, JAD. Associação entre exposição ao material particulado e internações por doenças respiratórias em crianças. Revista de Saúde Pública 2013;(47): 1209-1212 p.

CONTROLE DA POLUIÇÃO DO AR - PRONAR. RESOLUÇÃO CONAMA N491/2018 Padrões de qualidade do ar. Brasília (Brasil): CONSELHO NACIONAL DO MEIO AMBIENTE - CONAMA 2018.

DAPPER SN, SPOHR C, ZANINI RR. Poluição do ar como fator de risco para a saúde: uma revisão sistemática no estado de São Paulo. Estudos avançados 2016; 30 (86):83-97.

d'AMATO G, ROTTEM M, DAHL R, BLAISS MS, RIDOLO E, CECCHI L, et al. Climate change, migration, and allergic respiratory diseases: an update for the allergist. World Allergy Organization Journal 2011;4(7):121-125.

DAMILANO DCR, JORGE MPPM. Estudo da influência da poluição atmosférica e das condições meteorológicas na saúde em São José dos Campos. São Paulo 2006. In: SICINPE. 44.

EVO CPR, ULRYCH BK, TAKEGAWA B, SOARES G, NOGUEIRA G, de OLIVEIRA LO, et al. Poluição do ar e internação por insuficiência cardíaca congestiva em idosos no município de Santo André. Arquivos Brasileiros de Ciências da Saúde. [Internet]. 2011. [cited 15 fev 2017], 36(1):6-9 Disponível em: http://files.bvs.br/upload/S/19832451/2011/v36n1/a1917.pdf.

HAIR JF, ANDERSON RE, TATHAM RL, BLACK WC. Multivariate data analysis. 4 st ed. New Jersey: Prentice Hall, 1998. 
INSTITUTO BRASILEIRO DE GEOGRAFIA E ESTATÍSTICA - IBGE. [Internet] Banco de dados [citado 01 fevereiro 2016]. Disponível em: http://www.ibge.gov.br/home/.

KAISER HF. [The application of electronic computers to factor analysis]. Educational and Psychological Measurement 1960;20(1):141-151. Inglês.

KING G, POWELL EN. How not to lie without statistics. [Internet]. In: delivery at the 2008 Annual Meeting of the American Political Science Association. [Internet]. 2008 [citado 21 julho 2016]. Disponível em: http://gking.harvard.edu/files/mist.pdf

MINISTÉRIO DO MEIO AMBIENTE CONSELHO NACIONAL DO MEIO AMBIENTE Resolução Nº 3/1990. Controle da poluição do ar. (Brasil): CONAMA; De 1990.

MINISTÉRIO DO MEIO AMBIENTE. [Internet]. CIDADES SUSTENTÁVEIS. Qualidade do Ar, [citado 23 de outubro2016]. Disponível em: http://www.mma.gov.br/cidadessustentaveis/qualidade-do-ar.

NARDOCCI AC, FREITAS CUD, de LEON P, MONTEIRO AC, JUNGER W L, GOUVEIA NDC. Poluição do ar e doenças respiratórias e cardiovasculares: estudo de séries temporais em Cubatão. Cadernos de Saúde Pública 2013;29: 1867-76 p.

ORGANIZAÇÃO MUNDIAL DA SAÚDE - OMS. Diretrizes gerais e guia orientativo para elaboração de Análise de Impacto Regulatório - AIR / Subchefia de Análise e Acompanhamento de Políticas Governamentais [Internet] 2018 [citado 20 de junho2018]. Disponível em: https://www.gov.br/planalto/pt-br/acaogovernamental/politica-degovernanca/DiretrizesGeraiseGuiaOrientativo_final_27.09.2018.pdf.

PAIVA RF. Morbidade hospitalar por doenças associadas à poluição do ar na cidade de Volta Redonda, Rio de Janeiro: casos e custo econômico. Caderno de Saúde Coletiva 2014;22(2): 127-32.

ROMAN VVL, de CARVALHO Jr JA, NASCIMENTO LF, CESAR ACG. Efeitos de poluentes do ar e doenças respiratórias utilizando dados estimados por modelo matemático. Revista Ambiente e Água. 2015;10(4): 825-831.

SANTIAGO A, PRADO RJ, MODESTO FILHO P, ALONSO RV. Caracterização do material particulado suspenso no ar de Cuiabá-MT no período de queimadas. Matéria [Internet]. 2015 [cited 18 out 2018]; 20(1): 273-283, Available from: http://www.scielo.br/scielo.php?script=sci_arttext\&pid=S151770762015000100025\&ln $\mathrm{g}=\mathrm{en} \& \mathrm{nrm}=\mathrm{iso}$.

SILVA AMCD, MATTOS IE, IGNOTTI E, HACON SDS. Material particulado originário de queimadas e doenças respiratórias. Revista de Saúde Pública 2013;47(2): 345-52. 
TABACHNICK BG, FIDELL LS, ULLMAN JB. Using multivariate statistics. Boston, MA: Pearson. 2007. 330p.

TESTA JF. A poluição atmosférica por veículos automotores na Região Metropolitana de São Paulo: causas e impactos. Revista Eletrônica em Gestão, Educação e Tecnologia Ambiental Santa Maria [Internet]. 2015 [cited 26 out 2016]; 19(2): 1209-1221. Available from: https://periodicos.ufsm.br/reget/article/view/16944/pdf.

TINO V. Utilização de análise de componentes principais na regulagem de máquinas de injeção plástica. [dissertação]. Rio de Janeiro: Universidade Federal do Rio de Janeiro/ UFRJ, 2005. 90p.

TUAN TS, VENANCIO TS, NASCIMENTO LFC. Poluentes atmosféricos e internações por pneumonia em crianças. Estudo ecológico de série temporal. São Paulo Med. J. 2015;133(5): 408-413.

VICINI L, SOUZA AM, SOUZA FM. Técnicas Multivariadas Exploratórias: Teorias e Aplicações no SoftWare Statistica. Santa Maria/RS, Ed. UFSM, 2018. 240p.

WORLD HEALTH ORGANIZATION. Global health risks: mortality and burden of disease attributable to selected major risks. Geneva: World Health Organization 2009;(I): 62 p. 